Pacific Journal of Mathematics

ON NORMAL SUBGROUPS OF DIFFERENTIABLE 


\title{
ON NORMAL SUBGROUPS OF DIFFERENTIABLE HOMEOMORPHISMS
}

\author{
JAMES V. WHITTAKER
}

\begin{abstract}
The algebraic side of the group of all homeomorphisms of an interval or a circle has been studied exhaustively. In this paper the objects of study are the homeomorphisms with local polynomial approximations at each point. The algebraic side of the group of all such homeomorphisms is examined, particularly the minimal normal subgroup and the commutator subgroup. The results are like those in the topological case.
\end{abstract}

The normal subgroups of the group $H(X)$ of all homeomorphisms from a locally euclidean manifold $X$ onto itself have been studied by various authors for nearly forty years. The minimal normal subgroup of $H(X)$ has long been known to be the group $S H(X)$ generated by the members of $H(X)$ that are the identity outside some euclidean ball, and the manner of proof shows us also that $S H(X)$ has no proper normal subgroups of its own. When these same questions are asked of the group $C D_{n}(X)$ of all $n$-times continuously differentiable homeomorphisms, then the answers are much harder to come by. Recently D. B. A. Epstein [1] showed that the minimal normal subgroup and the commutator subgroup $C_{n}$ of $S C D_{n}(X)$ are one and the same and that $C_{1}$ is dense in $S C D_{1}(X)$. It is now known that $C_{n}=S C D_{n}(X)$ when $X$ is any euclidean space from some work of W. Thurston to appear.

In this paper we shall take $X$ to be 1-dimensional and study not $C D_{n}(X)$ but the group $E_{n}(X)$ of all homeomorphisms with local polynomial approximations of degree $n$ at each point of $X$. The minimal normal subgroups and the commutator subgroups of $E_{n}(X)$ and $S E_{n}(X)$ are exhibited, and $S E_{n}(X)$ is shown to have no proper normal subgroups of its own. The results are just like those in [2] for $H(X)$ and $S H(X)$.

2. Diffeomorphisms. Let $X$ be the line $E^{1}$ or the circle $S^{1}$ with local coordinate systems, and $H(X)$ the group of all homeomorphisms from $X$ onto itself. We shall single out certain subgroups of $H(X)$, writing $h^{\prime}$ for the first and $h^{(n)}$ for the $n$th derivative of some $h$ in $H(X)$. For each integer $n \geqq 1$ we put

$$
\begin{gathered}
D_{n}(X)=\left\{h \in H(X): h^{\prime}(x) \neq 0 \text { and } h^{(n)}(x) \text { exists for each } x \text { in } X\right\}, \\
C D_{n}(X)=\left\{h \in D_{n}(X): h^{(n)} \text { is continuous }\right\},
\end{gathered}
$$




$$
D_{\infty}(X)=\bigcap\left\{D_{n}(X): n \geqq 1\right\}=\bigcap\left\{C D_{n}(X): n \geqq 1\right\} .
$$

Evidently $D_{n}(X), C D_{n}(X)$, and $D_{\infty}(X)$ are groups.

A somewhat different family $E_{n}(X)$ consists of those $h$ in $H(X)$ which can be written as

$$
h(p+x)=h(p)+\sum_{i=1}^{n} a_{i} x^{i}+x^{n} e(x),
$$

where $p$ is any point in $X, U_{p}$ is a neighborhood of $0, a_{1} \neq 0$, and $e(x) \rightarrow 0$ as $x \rightarrow 0$. The coefficients $\alpha_{i}$ will depend upon $p$. Evidently $C D_{n}(X) \subset E_{n}(X)$. To see that $E_{n}(X)$ is a group, we will need the help of a lemma.

LEMMA 1. Given the integer $n \geqq 1$ and the polynomial $P(x)$ $a_{1} x+a_{2} x^{2}+\cdots+a_{n} x^{n}$ with $a_{1} \neq 0$, we can find a polynomial $Q(y)=b_{1} y+b_{2} y^{2}+\cdots+b_{n} y^{n}$ with $b_{1} \neq 0$ satisfying

$$
\lim _{x \rightarrow 0} \frac{x-Q(P(x))}{x^{n}}=0 \text {. }
$$

Proof. The equation $y=P(x)$ defines $x$ as an implicit function of $y$ in some neighborhood $V$ of $y=0$, and we can compute its Taylor series with remainder. Thus

$$
x=b_{0}+b_{1} y+b_{2} y^{2}+\cdots+b_{n} y^{n}+y^{n} R(y),
$$

where $R(y) \rightarrow 0$ as $y \rightarrow 0$. Now $b_{0}=0$ and

$$
1=P^{\prime}(x) x^{\prime}(y)=P^{\prime}(0) x^{\prime}(0)=a_{1} x^{\prime}(0),
$$

so that $b_{1} \neq 0$ and we can solve for $x^{(i)}(0)$, hence also $b_{i}$, by repeated differentiation. If we put $Q(y)=b_{1} y+b_{2} y^{2}+\cdots+b_{n} y^{n}$, then

$$
\begin{aligned}
& \lim _{x \rightarrow 0} \frac{x-Q(P(x))}{x^{n}}=\lim _{x \rightarrow 0} \frac{(P(x))^{n} R(P(x))}{x^{n}} \\
& =a_{1}^{n} \lim _{x \rightarrow 0} R(P(x))=0
\end{aligned}
$$

which is the result that we seek.

LEMma 2. The family $E_{n}(X)$ is a group.

Proof. Given some $h$ in $H(X)$ satisfying (1), we put $h(p)=q$ and $h(p+x)=q+y$. Thus (1) reads

$$
y=\sum_{i=1}^{n} a_{i} x^{i}+x^{n} e(x)=P(x)+x^{n} e(x), \quad x \in U_{p} .
$$

We let $Q(y)$ be the polynomial from Lemma 1 and define $d(y)$ by the 
equation

$$
x=Q(y)+y^{n} d(y),
$$

where $V_{q}$ is a neighborhood of 0 inside the $V$ from Lemma 1 and so small that $x$ lies in $U_{p}$. Now

$$
\begin{aligned}
\lim _{y \rightarrow 0} d(y) & =\lim _{y \rightarrow 0} \frac{x-Q(y)}{y^{n}}=\lim _{y \rightarrow 0} \frac{x-Q\left(P(x)+x^{n} e(x)\right)}{y^{n}} \\
& =\lim _{x \rightarrow 0} \frac{x^{n}}{y^{n}} \cdot \frac{x-Q(P(x))-b_{1} x^{n} e(x)-x^{n} S(x)}{x^{n}} \\
& =b_{1}^{n} \lim _{x \rightarrow 0}\left(\frac{x-Q(P(x))}{x^{n}}-b_{1} e(x)-S(x)\right)=0,
\end{aligned}
$$

where $S(x) \rightarrow 0$ as $x \rightarrow 0$. This means that $h^{-1}$ belongs to $E_{n}(X)$. Suppose next that $g$ belongs to $E_{n}(X)$ and

$$
g(q+y)=g(q)+\sum_{i=1}^{n} c_{i} y^{i}+y^{n} \bar{e}(y), \quad y \in V_{q},
$$

where $q$ is any point in $X, V_{q}$ is a neighborhood of $0, c_{1} \neq 0$, and $\bar{e}(y) \rightarrow 0$ as $y \rightarrow 0$. If we put $g(q)=r$ and $g(q+y)=r+z$, then (2) reads

$$
z=\sum_{i=1}^{n} c_{i} y^{i}+y^{n} \bar{e}(y)=R(y)+y^{n} \bar{e}(y), \quad y \in V_{q} .
$$

We define $\bar{d}(x)$ by the equation

$$
z=R(P(x))+x^{n} \bar{d}(x),
$$

$x \in W_{r}$,

where $W_{r}$ is a neighborhood of 0 inside $U_{p}$ and so small that $y$ lies in $V_{q}$. Now

$$
\begin{aligned}
\lim _{x \rightarrow 0} \bar{d}(x) & =\lim _{x \rightarrow 0} \frac{z-R(P(x))}{x^{n}} \\
& =\lim _{x \rightarrow 0} \frac{R\left(P(x)+x^{n} e(x)\right)+y^{n} \bar{e}(y)-R(P(x))}{x^{n}} \\
& =\lim _{x \rightarrow 0} \frac{c_{1} x^{n} e(x)+x^{n} T(x)}{x^{n}}+a_{1}^{n} \lim _{y \rightarrow 0} \bar{e}(y)=0,
\end{aligned}
$$

where $T(x) \rightarrow 0$ as $x \rightarrow 0$. This means that $g h$ belongs to $E_{n}(X)$, and $E_{n}(X)$ is a group.

Lemma 3. If $p$ is a limit point of the set $K(h)$ of all fixed points of $h$, then $P(x)=x$.

Proof. We are given a sequence $\left\{x_{m}\right\}$ converging to 0 , where 
$x_{m} \neq 0, h\left(p+x_{m}\right)=p+x_{m}$ for each $m$, and of course $h(p)=p$. Putting $x=x_{m}$ in (1), we have

$$
x_{m}=a_{1} x_{m}+a_{2} x_{m}^{2}+\cdots+a_{n} x_{m}^{n}+x_{m}^{n} e\left(x_{m}\right) .
$$

If we divide out by $x_{m}$ and let $m \rightarrow \infty$, then we get $a_{1}=1$. If we repeat this process, then we get $a_{2}=\cdots=a_{n}=0$. Therefore, $P(x)=x$.

Lemma 4. If $P(x)=x$ for every $p$ in $X$, then $h$ is a translation of the line or a rotation of the circle.

Proof. We have

$$
h^{\prime}(p)=\lim _{x \rightarrow 0} \frac{h(p+x)-h(p)}{x}=\lim _{x \rightarrow 0} \frac{x+x^{n} e(x)}{x}=1
$$

for each $p$ in $X$.

Evidently $E_{n}(X) \supset E_{n+1}(X)$, for we can write

$$
\begin{aligned}
h(p+x) & =h(p)+\sum_{i=1}^{n+1} a_{i} x^{i}+x^{n+1} e(x) \\
& =h(p)+\sum_{i=1}^{n} a_{i} x^{i}+x^{n}\left(a_{n+1} x+x e(x)\right),
\end{aligned}
$$

where $d(x)=a_{n+1} x+x e(x) \rightarrow 0$ as $x \rightarrow 0$. We put $E_{\infty}(X)=\bigcap\left\{E_{n}(X)\right.$ : $n \geqq 1\}$ and note that $E_{\infty}(X) \supset D_{\infty}(X)$.

Lemma 5. Suppose that

$$
\begin{aligned}
h(p+x) & =h(p)+\sum_{i=1}^{n} a_{i} x^{i}+x^{n} e(x) \\
& =h(p)+\sum_{i=1}^{n+1} \bar{a}_{i} x^{i}+x^{n+1} \bar{e}(x),
\end{aligned}
$$

Then $a_{i}=\bar{a}_{i}$ for $1 \leqq i \leqq n$.

Proof. We have

$$
\sum_{i=1}^{n} a_{i} x^{i}+x^{n} e(x)=\sum_{i=1}^{n+1} \bar{a}_{i} x^{i}+x^{n+1} \bar{e}(x), \quad x \in U_{p} .
$$

If we divide out by $x$ and let $x \rightarrow 0$, then we get $a_{1}=\bar{a}_{1}$. If we repeat this operation, then we get $a_{2}=\bar{a}_{2}, \cdots, a_{n}=\bar{a}_{n}$.

Lemma 5 shows us the form of a member $h$ of $E_{\infty}(X)$. In some neighborhood of each point $p$ we can write $h$ as a partial sum of a certain power series with a remainder that goes to zero faster than the highest power of $x$ retained, and the neighborhood depends on this power. 
From the simple fact that $D_{1}(X)=E_{1}(X)$ we are led to enquire whether $D_{n}(X)=E_{n}(X)$ for other values of $n$. To see that this is not so, we choose $h$ in $H(X)$ so that $h\left(p_{0}\right)=p_{0}$ at some point $p_{0}$ in $X$,

$$
h\left(p_{0}+x\right)=p_{0}+x+x^{3} \sin 1 / x
$$

for $x$ in a sufficiently small neighborhood to the right of $0, h(p)=p$ for $p$ in some neighborhood to the left of 0 , and $h$ beyond these neighborhoods has derivatives of all orders with first derivative that is never zero. Evidently $h$ belongs to $E_{2}(X)$. But $h^{\prime}\left(p_{0}\right)=1$,

$$
h^{\prime}\left(p_{0}+x\right)=1-x \cos 1 / x+3 x^{2} \sin 1 / x,
$$

and $h^{\prime \prime}\left(p_{0}\right)$ is not defined. Thus $h$ does not belong to $D_{2}(X)$, and $E_{2}(X)$ does not lie in $D_{2}(X)$. In the same way we can show that $E_{2 n}(X)$ does not lie in $D_{n+1}(X)$ when we put

$$
h\left(p_{0}+x\right)=p_{0}+x+x^{2 n+1} \sin 1 / x .
$$

If we consider the homeomorphism $g$ given in part by

$$
g\left(p_{0}+x\right)=p_{0}+x+x^{2} \sin 1 / x,
$$

then we find that $g$ belongs to $E_{1}(X)$ but not to $C D_{1}(X)$, and $E_{1}(X)$ does not lie in $C D_{1}(X)$. In the same way we can show that $E_{2 n-1}(X)$ does not lie in $C D_{n}(X)$ when we put

$$
g\left(p_{0}+x\right)=p_{0}+x+x^{2 n} \sin 1 / x .
$$

Finally, if we choose the homeomorphism $f$ given in part by

$$
f\left(p_{0}+x\right)=p_{0}+x+e^{-1 / x} \sin e^{1 / x},
$$

then $f$ evidently belongs to $E_{\infty}(X)$. But

$$
f^{\prime}\left(p_{0}+x\right)=1-\frac{\cos e^{1 / x}}{x^{2}}+\frac{e^{-1 / x} \sin e^{1 / x}}{x^{2}}
$$

shows us that $f^{\prime}\left(p_{0}+x\right)$ has no limit as $x \rightarrow 0$. Thus $f$ does not belong to $C D_{1}(X)$, and $E_{\infty}(X)$ does not lie in $C D_{1}(X)$. In particular, $D_{\infty}(X)$ is a proper subset of $E_{\infty}(X)$.

3. Minimal normal subgroups. We say that a member $h$ of $H(X)$ has compact support if $X-K(h)$ lies in a compact set different from $X$. For any subset $G$ of $H(X)$, we let $S G$ be the group generated by those members of $G$ with compact support. If $G$ is a group, then $S G$ is evidently a normal subgroup of $G$. We note that if $U, V$ are open subsets of $X$, and $U$ lies in a compact set different from $X$, then some member of $S D_{\infty}(X)$ maps $U$ into $V$. 
THEOREM 1. If $G$ is a subgroup of $H(X), S E_{n}(X)$ lies in the normalizer of $G$, and $G$ has more than one member, then $G \supset S E_{n}(X)$.

Proof. Suppose that $g_{0}$ belongs to $G$ and is not the identity. Then $g_{0}$ moves some point $p_{0}$ in $X$, and we can find a neighborhood $U_{0}$ of $p_{0}$ which does not meet $g_{0}\left(U_{0}\right)$. We put

$$
h_{0}\left(p_{0}+x\right)=p_{0}+x-e^{-1 / x}=p_{0}+x+S(x), \quad 0 \leqq x \leqq u,
$$

where $u$ is chosen so small that $h_{0}^{\prime}(p)>0$ for $p_{0}<p<p_{0}+u=p_{1}$ and $\left(p_{0}, p_{1}+u\right) \subset U_{0}$. Since $h_{0}(p)<p$ there, the sequence $\left\{p_{k}\right\}$ given by $p_{k+1}=h_{0}^{k}\left(p_{1}\right)$ will converge monotonely to $p_{0}$. We can define $h_{0}$ on the rest of $X$ so as to be supported on $\left(p_{0}, p_{1}+u\right)$ and belong to $E_{n}(X)$, for $S(x) / x^{n} \rightarrow 0$ as $x \rightarrow+0$ tells us that $h_{0}$ satisfies (1) to the right of $p=p_{0}$ with $P(x)=x$. Evidently $g_{0}^{-1} h_{0}^{-1} g_{0} h_{0}=g_{1}$ agrees with $h_{0}$ on $U_{0}$ and belongs to $G$. Suppose that $f$ is any member of $S E_{n}(X)$, and $X-K(f)=U$ lies in a compact set different from $X$. If we choose $f_{0}$ in $S D_{\infty}(X)$ so that $f_{0}(U) \subset\left(p_{2}, p_{1}\right)$, then $f_{0} f f_{0}^{-1}=f_{1}$ is supported on $\left(p_{2}, p_{1}\right)$ and belongs to $S E_{n}(X)$. If we can show that $f_{1}$ belongs to $G$, then $f$ must also belong to $G$, and $G \supset S E_{n}(X)$. We first note that

$$
g_{1}^{k}\left(\left[p_{2}, p_{1}\right]\right)=\left[p_{k+2}, p_{k+1}\right], \quad k=0,1,2, \cdots \text {. }
$$

We then define

$$
h(p)=\left\{\begin{array}{l}
g_{1}^{k} f_{1} g_{1}^{-k}(p) \text { for } p \text { in }\left[p_{k+2}, p_{k+1}\right], \quad k=0,1,2, \cdots, \\
p \text { for } p \text { in } X-\left(p_{0}, p_{1}\right] .
\end{array}\right.
$$

Lemma 3 tells us that we can join two pieces of $h$ together at $p=p_{k}$ so as to satisfy (1) there, for $e(x)$ may be defined in different ways on either side of $p$. Thus (1) holds at every point $p$ of $X$ except possibly $p=p_{0}$. To see that $h$ satisfies (1) at $p=p_{0}$, we observe that if $p$ lies in $\left[p_{k+2}, p_{k+1}\right]$, then so does $h(p)$. If we put $p_{k}=p_{0}+x_{k}$, then

$$
\begin{aligned}
& \lim _{x \rightarrow 0} \frac{\left|h\left(p_{0}+x\right)-p_{0}-x\right|}{x^{n}} \leqq \lim _{k \rightarrow \infty} \frac{p_{k+1}-p_{k+2}}{\left(p_{k+2}-p_{0}\right)^{n}} \\
& \quad=\lim _{k \rightarrow \infty} \frac{p_{k+1}-h_{0}\left(p_{k+1}\right)}{\left(h_{0}\left(p_{k+1}\right)-p_{0}\right)^{n}}=\lim _{k \rightarrow \infty} \frac{-S\left(x_{k+1}\right)}{\left(x_{k+1}+S\left(x_{k+1}\right)\right)^{n}} \\
& \quad=\lim _{k \rightarrow \infty} \frac{-S\left(x_{k+1}\right) / x_{k+1}^{n}}{\left(1+S\left(x_{k+1}\right) / x_{k+1}\right)^{n}}=0 .
\end{aligned}
$$

This means that $h$ satisfies (1) at $p=p_{0}$ with $P(x)=x$, and so $h$ belongs to $S E_{n}(X)$. Now $g_{1} h g_{1}^{-1}$ is supported on $\left(p_{0}, p_{2}\right)$ and agrees with $h$ there, for if $p$ lies in $\left[p_{k+2}, p_{k+1}\right]$ and $k \geqq 1$, then

$$
g_{1} h g_{1}^{-1}(p)=g_{1} g_{1}^{k-1} f_{1} g_{1}^{-k+1} g_{1}^{-1}(p)=g_{1}^{k} f_{1} g_{1}^{-k}(p)=h(p) .
$$


Therefore, $h g_{1} h^{-1} g_{1}^{-1}=f_{1}$ must lie in $G$, and our proof is complete.

COROLlary 1. Theorem 1 remains true if we replace $n$ by $\infty$.

Proof. We have already seen that $h_{0}$ satisfies (1) to the right of $p=p_{0}$ with $P(x)=x$ for every value of $n$. Hence, we can define $h_{0}$ on the rest of $X$ so as to be supported on $\left(p_{0}, p_{1}+u\right)$ and belong to $E_{\infty}(X)$. The rest of the proof is the same as before with $n$ replaced by $\infty$.

Corollary 2. $S E_{n}(X)$ and $S E_{\infty}(X)$ are simple groups.

CoRollary 3. $S D_{1}(X)$ is a simple group.

Proof. Evidently $E_{1}(X)=D_{1}(X)$.

CoRollary 4. The commutator subgroup of $S E_{n}(X)$ is $S E_{n}(X)$, and the same for $S E_{\infty}(X)$.

Proof. Some commutators are evidently not the identity.

For any subset $G$ of $H(X)$, we write $G^{+}$for the family of all orientation-preserving members of $G$, and $G^{-}$for $G-G^{+}$. If $G$ is a group, then $G^{+}$is evidently a normal subgroup of $G$ with index one or two.

Corollary 5. If $X=S^{1}$, then $E_{n}^{+}(X)$ and $E_{\infty}^{+}(X)$ are simple groups.

Proof. It is enough to show that $E_{n}^{+}(X)=S E_{n}(X)$. To see this, we use the same method as for the case $H^{+}(X)=S H(X)$. Given $h$ in $E_{n}^{+}(X)$, we can piece together a member $g$ of $E_{n}^{+}(X)$ that agrees with $h$ on a small open subset of $X$ and agrees with the identity on another small open subset of $X$. Then $g$ and $g^{-1} h$ both belong to $S E_{n}(X)$, and so does $g\left(g^{-1} h\right)=h$. The same argument works for $E_{\infty}^{+}(X)$.

4. Behavior at the endpoints. From now on we shall deal only with the space $X=[-1,+1]$ and think of $E^{1}$ as the subset $(-1,+1)$ of $X$. For any subset $G$ of $H(X)$ we put

$$
\begin{aligned}
& S_{-} G=\{h \in G: K(h) \supset[-1, p) \text { for some } p>-1\}, \\
& S_{+} G=\{h \in G: K(h) \supset(p,+1] \text { for some } p<+1\} .
\end{aligned}
$$

Evidently $S_{-} G \cap S_{+} G=S G$, and both are normal subgroups of $G$ 
provided that $G$ is a subgroup of $H^{+}(X)$. Thus $S_{-} E_{n}(X), S_{+} E_{n}(X)$ are normal subgroups of $E_{n}^{+}(X)$, and the same is true if we replace $n$ by $\infty$. The normal subgroups of $H^{+}(X)$ are few in number, namely $S_{-} H(X), S_{+} H(X)$, and $S H(X)$. Those of $H(X)$ are even fewer, namely $H^{+}(X)$ and $S H(X)$. Proofs are given in [2]. On the other hand, those of $E_{n}^{+}(X), E_{\infty}^{+}(X), E_{n}(X)$, and $E_{\infty}(X)$ are very numerous. Our aim in the rest of this paper is to show how some of them may be constructed.

The behavior of a member $h$ of $E_{n}(X)$ at $p=-1$ or +1 is described by (1), namely

$$
\begin{array}{ll}
h(-1+x)=h(-1)+P_{-}(x)+x^{n} d(x), & x \in U_{-1}, \\
h(+1+x)=h(+1)+P_{+}(x)+x^{n} e(x), & x \in U_{+1},
\end{array}
$$

where $d(x), e(x) \rightarrow 0$ as $x \rightarrow 0, U_{-1}=[0,+u), U_{+1}=(-u, 0]$, and $u>0$. Let $\Delta_{n-}(X)$ be the family of those $h$ in $E_{n}(X)$ for which $P_{-}(x)=x$, $\Delta_{n+}(X)$ the family for which $P_{+}(x)=x$, and $\Delta_{n-}(X) \cap \Delta_{n+}(X)=\Delta_{n}(X)$. Evidently all three are normal subgroups of $E_{n}^{+}(X)$. To see that $\Delta_{n}(X)$ is also a normal subgroup of $E_{n}(X)$, we have to check that $f \Delta_{n}(X) f^{-1} \subset \Delta_{n}(X)$ for each $f$ in $E_{n}^{-}(X)$. Now $f(+1)=-1=f^{-1}(+1)$, and Lemma 2 says that $f$ satisfies (1) with $P_{-}\left(P_{+}(x)\right)=x=P_{+}\left(P_{-}(x)\right)$. Thus $f h f^{-1}$ belongs to $\Delta_{n}(X)$ whenever $h$ does, and $\Delta_{n}(X)$ is normal in $E_{n}(X)$. Lemma 3 tells us that if $h$ belongs to $E_{n}(X)$, and the derived set of $K(h)$ includes +1 , then $h$ belongs to $\Delta_{n+}(X)$, whereas if it includes -1 , then $h$ belongs to $\Delta_{n-}(X)$. These results are still valid when we replace $n$ by $\infty$, where $P_{+}(x)$ and $P_{-}(x)$ are now power series in $x$.

Other normal subgroups of $E_{n}(X)$ can also be constructed. Choose a sequence $\left\{p_{k}\right\}$ converging to -1 , where $p_{k}=-1+x_{k}, 0<x_{k}<+1$, $x_{k+1} / x_{k} \rightarrow L$ as $k \rightarrow \infty$, and $0 \leqq L<1$. Then choose a sequence $\left\{q_{k}\right\}$ converging to -1 , where $q_{k}=p_{k}+y_{k}, 0<y_{k}<+1$, and $y_{k} / x_{k}^{m} \rightarrow 0$ as $k \rightarrow \infty$ for every $m$. We define a function $g$ in $H(X)$ so that $X-K(g)=\bigcup\left\{\left(p_{k}, q_{k}\right)\right\}, g$ satisfies (1) at every point $p$ in $X$ except possibly $p=-1$, and $P(x)=x$ at $p=p_{k}$ or $q_{k}$. To see that (1) holds at $p=-1$, we note that if $x$ lies in $\left(p_{k}, q_{k}\right)$, then so does $g(x)$. Thus

$$
\lim _{x \rightarrow+0} \frac{|g(-1+x)-g(-1)-x|}{x^{n}} \leqq \lim _{k \rightarrow \infty} \frac{y_{k}}{x_{k}^{n}}=0,
$$

and (1) holds with $P_{-}(x)=x$. Since $P_{+}(x)=x$, we have $g$ in $\Delta_{n}(X)$. The normal subgroup $G$ of $E_{n}(X)$ generated by $g$ consists of products of conjugates in $E_{n}(X)$ of $g$, and $G$ is clearly larger than $S E_{n}(X)$. To see that $G$ is a proper subgroup of $\Delta_{n}(X)$, we will show that for every member $f$ of $G$, the derived set of $K(f)$ includes -1 . The same argument shows that it includes +1 as well. If $h$ belongs to $E_{n}(X)$ 
and satisfies (1) at $p=-1$ with $P(x)=P_{-}^{*}(x)=a_{1} x+\cdots$, then we shall put $h\left(p_{k}\right)=h(-1)+x_{k}^{*}$ and $h\left(q_{k}\right)=h\left(p_{k}\right)+y_{k}^{*}$. We have

$$
\lim _{k \rightarrow \infty} \frac{x_{k+1}^{*}}{x_{k}^{*}}=\lim _{k \rightarrow \infty} \frac{P_{-}^{*}\left(x_{k+1}\right)+x_{k+1}^{n} e^{*}\left(x_{k+1}\right)}{P_{-}^{*}\left(x_{k}\right)+x_{k}^{n} e^{*}\left(x_{k}\right)}=\lim _{k \rightarrow \infty} \frac{x_{k+1}}{x_{k}}=L,
$$

(3) $\lim _{k \rightarrow \infty} \frac{y_{k}^{*}}{\left(x_{k}^{*}\right)^{n}}=\lim _{k \rightarrow \infty} \frac{P_{-}^{*}\left(x_{k}+y_{k}\right)-P_{-}^{*}\left(x_{k}\right)+\left(x_{k}+y_{k}\right)^{n} e^{*}\left(x_{k}+y_{k}\right)-x_{k}^{n} e^{*}\left(x_{k}\right)}{\left(P_{-}^{*}\left(x_{k}\right)+x_{k}^{n} e^{*}\left(x_{k}\right)\right)^{n}}$

$$
=\lim _{k \rightarrow \infty} \frac{a_{1} y_{k}}{a_{1}^{n} x_{k}^{n}}=0 \text {. }
$$

Suppose that $f$ is the product of $j$ conjugates $g_{i}=h_{i} g h_{i}^{-1}(1 \leqq i \leqq j)$ of $g$, where $f=g_{j} \cdots g_{1}$. Our work would be made easier if any $h_{i}$ lay in $E_{n}^{-}(X)$, for then $K\left(g_{i}\right)=h_{i}(K(g))$ would include a neighborhood of -1 . So we shall assume that $h_{i}$ lies in $E_{n}^{+}(X)$ for $1 \leqq i \leqq j$. We put

$$
\begin{aligned}
& p_{k}^{(i)}=h_{i}\left(p_{k}\right)=h_{i}(-1)+x_{k}^{(i)}, \\
& q_{k}^{(i)}=h_{i}\left(q_{k}\right)=h_{i}\left(p_{k}\right)+y_{k}^{(i)},
\end{aligned}
$$$$
1 \leqq i \leqq j
$$

From (3) we know that

$$
\lim _{k \rightarrow \infty} x_{k+1}^{(i)} / x_{k}^{(i)}=L, \lim _{k \rightarrow \infty} y_{k}^{(i)} /\left(x_{k}^{(i)}\right)^{n}=0 .
$$

Given any number $u>0$, we choose $k_{0}$ so large that $k \geqq k_{0}$ implies

$$
x_{k+1}^{(i)} / x_{k}^{(i)} \leqq L^{*}<1, \quad y_{k}^{(i)} / x_{k}^{(i)} \leqq\left(1-L^{*}\right) / 2 j, \quad 1 \leqq i \leqq j,
$$

and put

$$
k(i)=\sup \left\{k: x_{k}^{(i)}>u \text { or } k=k_{0}\right\}, \quad 1 \leqq i \leqq j .
$$

From $K\left(g_{i}\right)=h_{i}(K(g))$ and $K(f) \supset \bigcap\left\{K\left(g_{i}\right): 1 \leqq i \leqq j\right\}$ we see that

(4) $(X-K(f)) \bigcap[-1,-1+u) \subset \bigcup\left\{\left(p_{k}^{(i)}, q_{k}^{(i)}\right): k>k(i), 1 \leqq i \leqq j\right\}$.

Now the sum of the lengths of all the intervals on the right side of (4) is

$$
\begin{aligned}
& \sum_{i=1}^{j} \sum_{k=k(i)+1}^{\infty} y_{k}^{(i)} \leqq \sum_{i=1}^{j} \sum_{k=k(i)+1}^{\infty} \frac{1-L^{*}}{2 j} x_{k}^{(i)} \\
& \quad \leqq \frac{1-L^{*}}{2 j} \sum_{i=1}^{j} \sum_{m=0}^{\infty} x_{k(i)+1}^{(i)}\left(L^{*}\right)^{m}=\frac{1-L^{*}}{2 j} \sum_{i=1}^{j} \frac{x_{k(i)+1}^{(i)}}{1-L^{*}} \\
& \quad \leqq \frac{1}{2 j} \sum_{i=1}^{j} u=\frac{u}{2} .
\end{aligned}
$$

These intervals could not possibly cover $(-1,-1+u)$, for otherwise a finite chain of them would reach from $-1+u / 5$ to $-1+4 u / 5$, which is impossible. Hence, $X-K(f)$ does not contain $(-1,-1+u)$ for any $u>0$, and the derived set of $K(f)$ includes -1 . Therefore, $G$ 
is not $\Delta_{n}(X)$. Evidently the same argument also shows that we can make $g$ belong to $\Delta_{\infty}(X)$, and the normal subgroup of $E_{\infty}(X)$ generated by $g$ is neither $S E_{\infty}(X)$ nor $\Delta_{\infty}(X)$. We shall now sum up these results.

THEOREM 2. There are normal subgroups of $E_{n}(X)$ that lie between $S E_{n}(X)$ and $\Delta_{n}(X)$. The same is true of normal subgroups of $E_{\infty}(X)$ between $S E_{\infty}(X)$ and $\Delta_{\infty}(X)$.

We let $e$ be the identity element of $H(X)$ and call a member $h$ of $H(X)$ an involution if $h^{2}=e$. Evidently an involution different from $e$ has just one fixed point in $(-1,+1)$.

LEMma 6. Suppose that for $i=1,2$ there is an involution $h_{i}$ of $E_{n}(X)$ with fixed point $p_{i}$ that satisfies (1) at $p=p_{i}$ with $P(x)=P_{i}(x)$. If there is a polynomial $Q(x)=a_{1} x+a_{2} x^{2}+\cdots$ with $a_{1}>0$ such that $P_{1} Q(x)$ and $Q P_{2}(x)$ agree in all terms of degree $\leqq n$, then there is a member $f$ of $S_{-} E_{n}(X)$ such that $h_{1} f=f h_{2}$. We may also choose $f$ in $S_{+} E_{n}(X)$, and the result remains true if we replace $n$ by $\infty$.

Proof. We choose $h_{0}$ in $S E_{n}(X)$ so that $h_{0}\left(p_{2}\right)=p_{1}$ and $h_{0}$ satisfies (1) at $p=p_{2}$ with $P(x)=Q(x)$. We shall assume that $h_{i}$ is different from $e$, so that $h_{i}$ lies in $E_{n}^{-}(X)$. Thus $h_{3}=h_{0}^{-1} h_{1} h_{0}$ is an involution of $E_{n}(X)$ with fixed point $p_{2}$ and satisfying (1) at $p=p_{2}$ with $P(x)=$ $P_{2}(x)$. Our result will be proved if we can find $g$ in $S_{-} E_{n}(X)$ so that $h_{3} g=g h_{2}$. We put

$$
g(p)= \begin{cases}p & \text { for }-1 \leqq p \leqq p_{2} \\ h_{3} h_{2}(p) & \text { for } p_{2} \leqq p \leqq+1\end{cases}
$$

Evidently $g$ belongs to $S_{-} H(X)$ and satisfies (1) at each point $p$ of $X$, except possibly $p=p_{2}$. But $h_{2}^{2}=e$ satisfies (1) at $p=p_{2}$ with $P(x)=$ $P_{2}^{2}(x)=x$, so $h_{3} h_{2}$ also satisfies (1) at $p=p_{2}$ with $P(x)=x$. Consequently, $g$ belongs to $S_{-} E_{n}(X)$, and

$$
g h_{2}(p)= \begin{cases}h_{3} h_{2} h_{2}(p)=h_{3}(p)=h_{3} g(p) \text { for }-1 \leqq p \leqq p_{2} \\ h_{2}(p)=h_{3} h_{3} h_{2}(p)=h_{3} g(p) \text { for } p_{2} \leqq p \leqq+1\end{cases}
$$

Hence, $g h_{2}=h_{3} g$.

5. Groups of polynomials. It is now time to study the compositions of polynomials more closely. We begin with the family $\Pi_{\infty}$ of power series

$$
P(x)=a_{1} x+a_{2} x^{2}+\cdots+a_{n} x^{n}+\cdots, \quad a_{1} \neq 0
$$


with any coefficients whatever. By working with the partial sums of $P(x)$, we see that the composition $P Q(x)=P(Q(x))$ of two members $P, Q$ of $\Pi_{\infty}$ also belongs to $\Pi_{\infty}$, that $P_{0}(x)=x$ is the identity element of $\Pi_{\infty}$, and from Lemma 1 that $P$ has an inverse $P^{-1}$ in $\Pi_{\infty}$ satisfying $P^{-1} P=P_{0}$. Since the associative law always holds for composition, we see that $\Pi_{\infty}$ is a group. For each integer $n \geqq 1$, we define an equivalence relation $P \Xi_{n} Q$ between members $P$ and $Q$ of $\Pi_{\infty}$ to mean that $P(x)$ and $Q(x)$ agree in all terms of degree $\leqq n$. This relation is evidently compatible with the group operation, and the quotient group $\Pi_{n}$ we can think of as the group of all $n$th degree polynomials

$$
P(x)=a_{1} x+a_{2} x^{2}+\cdots+a_{n} x^{n}, \quad a_{1} \neq 0
$$

under composition. If $\Gamma$ is a subgroup of $\Pi_{\infty}$, then we shall let $\Gamma^{n}$ be the quotient group of $\Gamma$ under $\Xi_{n}$. We put $\left\{P_{0}\right\}=\Delta_{\infty}$ and use $\Delta_{n}$ for the subgroup of $\Pi_{n}$ with just one element. A normal subgroup of $\Pi_{\infty}$ closely related to $\Delta_{n}$ is the kernel $\Delta_{n}^{*}$ of the homomorphism from $\Pi_{\infty}$ onto $\Pi_{n}$ induced by the relation $\Xi_{n}$. It consists of all members of $\Pi_{\infty}$ whose first $n$ coefficients are $1,0, \cdots, 0$. We shall use $\Pi$ to mean either $\Pi_{\infty}$ or $\Pi_{n}$, and the same for $\Xi, \Delta$, and $E(X)$. The family of all those $P$ in $I$ with $a_{1}>0$ we will denote by $\Pi^{+}$and put $\Pi-\Pi^{+}=\Pi^{-}$. Evidently $\Pi^{+}$is a normal subgroup of $\Pi$ and compatible with $\Xi$. We define $\Gamma^{+}=\Gamma \cap \Pi^{+}, \Gamma^{-}=\Gamma-\Gamma^{+}$, and note that $\Gamma^{+}$is a normal subgroup of $\Gamma$ with index one or two.

LEMma 7. The only normal subgroups $\Gamma$ of $\Pi_{n}$ that lie in $\Delta_{1}^{* n}$ are $\Gamma=\Delta_{m}^{* n}$ for $1 \leqq m \leqq n$.

Proof. It is evidently sufficient to prove the following statement for each value of $m$ :

(5) For each integer $k$ with $0<k<m$, if $\Gamma$ contains some member $P$ of the form $P(x)=x+a_{k+1} x^{k+1}+\cdots$ with $a_{k+1} \neq 0$, then for each $R$ in $\Delta_{k}^{* n}, \Gamma$ contains some $Q$ such that $Q \Xi_{m} R$.

Now (5) certainly holds when $m=1$. We shall proceed by induction and suppose that (5) holds for some value $m>1$. To verify it for $m+1$, we first note that if $k=m$ and $P_{1}(x)=a_{1} x$, then

$$
\begin{aligned}
P^{-1}(x) & =x-a_{k+1} x^{k+1}+\cdots, \\
P_{1}^{-1} P P_{1}(x) & =x+a_{1}^{m} a_{m+1} x^{m+1}+\cdots, \\
P_{1}^{-1} P^{-1} P_{1}(x) & =x-a_{1}^{m} a_{m+1} x^{m+1}+\cdots .
\end{aligned}
$$

Evidently $P_{1}^{-1} P P_{1}$ and $P_{1}^{-1} P^{-1} P_{1}$ both belong to $\Gamma$, and by varying $a_{1}$ through positive values we get all coefficients of $x^{m+1}$. Thus (5) holds in this case. If $k<m$, then our induction hypothesis says that $\Gamma$ 
contains some member $Q$ of the form

$$
Q(x)=x+b_{m} x^{m}+b_{m+1} x^{m+1}+\cdots,
$$

where $b_{m} \neq 0$. If we put $P_{2}(x)=x+a_{2} x^{2}$, then

$$
Q P_{2}(x)=x+a_{2} x^{2}+b_{m} x^{m}+\left(b_{m+1}+m a_{2} b_{m}\right) x^{m+1}+\cdots,
$$

and this agrees with $P_{2} \bar{Q}(x)$ in all terms of degree $\leqq m+1$, where

$$
\bar{Q}(x)=x+b_{m} x^{m}+\left(b_{m+1}+(m-2) a_{2} b_{m}\right) x^{m+1}+\cdots .
$$

Thus $Q P_{2} \Xi_{m+1} P_{2} \bar{Q}$ and $P_{2}^{-1} Q P_{2} \Xi_{m+1} \bar{Q}$ while $P_{2}^{-1} Q P_{2}$ lies in $\Gamma$. If we put

$$
\begin{array}{ll}
P_{m}(x)=x+a_{m} x^{m}, & P_{m+1}(x)=x+a_{m+1} x^{m+1}, \\
Q_{m}(x)=x+c_{m} x^{m}, & Q_{m+1}(x)=x+c_{m+1} x^{m+1},
\end{array}
$$

then evidently $P_{i} Q_{j} \Xi_{m+1} Q_{j} P_{i}$ for $i, j=m, m+1$. We must now distinguish the cases $m>2$ and $m=2$. In the case $m>2$ we have

$$
P_{m}^{-1}(x)=x-a_{m} x^{m}+\cdots, \quad P_{m+1}^{-1}(x)=x-a_{m+1} x^{m+1}+\cdots
$$

for the first $m+1$ terms. If we put $a_{m}=b_{m}, c_{m}=b_{m}, a_{m+1}=b_{m+1}$, and $c_{m+1}=b_{m+1}+(m-2) a_{2} b_{m}$, then

$$
\begin{gathered}
Q \Xi_{m+1} P_{m} P_{m+1}, \quad \bar{Q} \Xi_{m+1} Q_{m} Q_{m+1}, \\
Q^{-1} \bar{Q} \Xi_{m+1} P_{m}^{-1} Q_{m} P_{m+1}^{-1} Q_{m+1} \Xi_{m+1} P_{m+1}^{-1} Q_{m+1},
\end{gathered}
$$

where

$$
P_{m+1}^{-1} Q_{m+1}(x)=x+(m-2) a_{2} b_{m} x^{m+1}+\cdots .
$$

Thus we get all coefficients of $x^{m+1}$ by varying $a_{2}$, and

$$
P_{m+1}^{-1} Q_{m+1} \Xi_{m+1} Q^{-1} P_{2}^{-1} Q P_{2}=R_{m+1},
$$

where $R_{m+1}$ lies in $\Gamma$. By our induction hypothesis, we can find a member of $\Gamma \cap \Delta_{k}^{* n}$ with any desired coefficients of $x^{k+1}, \cdots, x^{m}$. If we multiply this member on the left by $R_{m+1}$, then we keep the same coefficients of $x^{k+1}, \cdots, x^{m}$ and get any desired coefficient of $x^{m+1}$. This verifies (5) for $m+1$ when $m>2$. Finally, consider the case $m=2$. The argument for $k=m$ is still valid, so we need only consider $k=1$. Thus our induction hypothesis says that $\Gamma$ contains some member $Q$ of the form

$$
Q(x)=x+b_{2} x^{2}+b_{3} x^{3}+\cdots,
$$

where $b_{2} \neq 0$. We can check by induction that for any integer $i$,

$$
\begin{gathered}
P_{2}^{i}(x)=x+i a_{2} x^{2}+i(i-1) a_{2}^{2} x^{3}+\cdots, \\
P_{3}^{i}(x)=x+i a_{3} x^{3}+\cdots
\end{gathered}
$$


If we put $a_{2}=b_{2}$ and $a_{3}=b_{3}$, then $Q \Xi_{3} P_{2} P_{3}$. Evidently $P_{1}^{-1} Q P_{1}$ lies in $\Gamma$ and has the form

$$
P_{1}^{-1} Q P_{1}(x)=x+a_{1} b_{2} x^{2}+a_{1}^{2} b_{3} x^{3}+\cdots .
$$

If we put $c_{2}=a_{1} b_{2}$ and $c_{3}=a_{1}^{2} b_{3}$, then $P_{1}^{-1} Q P_{1} \Xi_{3} Q_{2} Q_{3}$. Finally, $Q^{i} P_{1}^{-1} Q P_{1}=R_{3}$ lies in $\Gamma$ and

$$
R_{3} \Xi_{3} P_{2}^{i} P_{3}^{i} Q_{2} Q_{3} \Xi_{3}\left(P_{2}^{i} Q_{2}\right)\left(P_{3}^{i} Q_{3}\right)
$$

Thus $R_{3}$ has the form

$$
R_{3}(x)=x+\left(i+a_{1}\right) b_{2} x^{2}+\left(i(i-1) b_{2}^{2}+\left(i+a_{1}^{2}\right) b_{3}\right) x^{3}+\cdots .
$$

If we choose $a_{1}=-i$, then

$$
R_{3}(x)=x+\left(\left(b_{2}^{2}+b_{3}\right) i^{2}-\left(b_{2}^{2}-b_{3}\right) i\right) x^{3}+\cdots .
$$

The coefficients $b_{2}^{2}+b_{3}$ and $b_{2}^{2}-b_{3}$ are not both zero, so we can choose some integer $i$ for which the coefficient of $x^{3}$ is not zero. As in the case $k=m$ above, we can find a member of $\Gamma$ with any coefficient of $x^{3}$, and the last part of the argument for $k<m$ completes the verification of (5) for the case $m=2$. Hence, our induction step is valid in every case.

THEOREM 3. Each normal subgroup $\Gamma$ of $\Pi_{n}$ is either $\Delta_{m}^{* n}$ with $1 \leqq m \leqq n$ or the inverse under the canonical mapping from $\Pi_{n}$ onto $\Pi_{1}$ of a subgroup $N$ of $\Pi_{1}$.

Proof. Each member $P$ of $\Gamma$ has the form

$$
P(x)=a_{1} x+a_{2} x^{2}+a_{3} x^{3}+\cdots, \quad a_{1} \neq 0 .
$$

Evidently $N=\Gamma^{1}$ is a subgroup of $\Pi_{1}$ which we shall identify with the subgroup of $\Pi_{n}$ consisting of all polynomials $P_{1}(x)=a_{1} x$. Evidently $\Pi_{1}$ is the group of nonzero real numbers under multiplication. Now $\Gamma \cap \Delta_{1}^{* n}$ is a normal subgroup of $\Pi_{n}$, and Lemma 7 says that $\Gamma \cap \Delta_{1}^{* n}=\Delta_{m}^{* n}$ with $1 \leqq m \leqq n$. If

$$
P(x)=a_{1} x+a_{k+1} x^{k+1}+\cdots, \quad a_{k+1} \neq 0,
$$

and if we put $Q_{1}(x)=b_{1} x$, then

$$
\begin{gathered}
P^{-1}(x)=\left(1 / a_{1}\right) x-\left(a_{k+1} / a_{1}^{k+2}\right) x^{k+1}+\cdots, \\
Q_{1}^{-1} P Q_{1}(x)=a_{1} x+a_{k+1} b_{1}^{k} x^{k+1}+\cdots, \\
P^{-1} Q_{1}^{-1} P Q_{1}(x)=x+\left(a_{k+1} / a_{1}\right)\left(b_{1}^{k}-1\right) x^{k+1}+\cdots .
\end{gathered}
$$

Since $P^{-1} Q_{1}^{-1} P Q_{1}$ belongs to $\Gamma$, we get $\Delta_{k}^{* n} \subset \Gamma$ by taking $b_{1} \neq \pm 1$. 
If we put $P_{1}(x)=a_{1} x$ as above, then $P_{1}^{-1} P$ belongs to $\Delta_{k}^{* n}$, and $P_{1}$ belongs to $\Gamma$. Thus $N$ lies in $\Gamma$. If we put $Q_{2}(x)=x+b_{2} x$, then $Q_{2}^{-1}(x)=x-b_{2} x^{2}+\cdots$ and

$$
\begin{aligned}
P_{1}^{-1} Q_{2}^{-1} P_{1} Q_{2}(x) & =\left(1 / a_{1}\right)\left(a_{1} x+\left(a_{1}-a_{1}^{2}\right) b_{2} x^{2}+\cdots\right) \\
& =x+\left(1-a_{1}\right) b_{2} x^{2}+\cdots .
\end{aligned}
$$

If we suppose that $N$ is more than the identity, then we can choose $a_{1} \neq 1, b_{2} \neq 0$, and get a member of $\Delta_{1}^{* n}-\Delta_{2}^{* n}$. Since $P_{1}^{-1} Q_{2}^{-1} P_{1} Q_{2}$ belongs to $\Gamma$, we must have $\Delta_{1}^{* n} \subset \Gamma$. Hence, for any $Q$ in $\Pi_{n}$ of the form $Q(x)=a_{1} x+\cdots$, we know that $P_{1}^{-1} Q$ belongs to $\Delta_{1}^{* n}$, and $Q$ belongs to $\Gamma$.

COROLLARY 1. Each normal subgroup $\Gamma$ of $\Pi_{n}^{+}$is either $\Delta_{m}^{* n}$ with $1 \leqq m \leqq n$ or the inverse under the canonical mapping from $\Pi_{n}^{+}$onto $\Pi_{1}^{+}$of a subgroup $N$ of $\Pi_{1}^{+}$.

Proof. The proofs of Lemma 7 and Theorem 3 apply here, for nowhere was the leading coefficient of any polynomial assumed to be negative.

COROLlaRY 2. Each normal subgroup $\Gamma$ of $\Pi_{n}^{+}$is normal in $\Pi_{n}$.

Proof. Evidently each subgroup $N$ of $\Pi_{1}^{+}$is normal in $\Pi_{1}$.

COROLlaRY 3. The group I generated by all involutions of $\Pi_{n}$ consists of all members of $\Pi_{n}$ with leading coefficient \pm 1 .

Proof. Since each conjugate of each involution of $\Pi_{n}$ is also an involution, $I$ must be a normal subgroup of $\Pi_{n}$. If $P$ belongs to $I$ and $P(x)=a_{1} x+\cdots$, then $P^{2}=P_{0}, a_{1}^{2}=1$, and $a_{1}= \pm 1$. Evidently $P(x)=-x$ belongs to $I$, so our result follows from Theorem 3 .

CoRollaRy 4. The commutator subgroup $K$ of $\Pi_{n}$ is $\Delta_{1}^{* n}$.

Proof. If $P(x)=a_{1} x+\cdots$ and $Q(x)=b_{1} x+\cdots$, then

$$
P^{-1} Q^{-1} P Q(x)=x+\cdots
$$

and $K$ lies in $\Delta_{1}^{* n}$. If we put

$$
P_{1}(x)=a_{1} x, \quad Q_{2}(x)=x+b_{2} x^{2},
$$

then

$$
\begin{gathered}
P_{1}^{-1}(x)=\left(1 / a_{1}\right) x, \quad Q_{2}^{-1}(x)=x-b_{2} x^{2}+\cdots, \\
P_{1}^{-1} Q_{2}^{-1} P_{1} Q_{2}(x)=x+\left(1-a_{1}\right) b_{2} x^{2}+\cdots .
\end{gathered}
$$


For $a_{1} \neq 1$ and $b_{2} \neq 0$ we get a member of $\Delta_{1}^{* n}-\Delta_{2}^{* n}$. Hence, $K=\Delta_{1}^{* n}$.

LEMMA 8. Every two involutions in $I I$ different from $P_{0}$ are conjugate.

Proof. It is evidently sufficient to show that every involution $P$ different from $P_{0}$ with

$$
P(x)=a_{1} x+a_{2} x^{2}+a_{3} x^{3}+\cdots
$$

is conjugate to $R_{\infty}$ with $R_{\infty}(x)=-x$. We know that $a_{1}= \pm 1$. If $a_{1}=+1$, then

$$
\begin{gathered}
P(x)=x+a_{k} x^{k}+\cdots, \\
x=P^{2}(x)=x+2 a_{k} x^{k}+\cdots
\end{gathered} \quad a_{k} \neq 0,
$$

which is impossible. Thus $a_{1}=-1$. Now suppose that for some integer $m \geqq 1$ we have found a member $P_{m}$ of $\Pi$ satisfying

$$
P_{m}^{-1} P P_{m}(x)=-x+a_{j} x^{j}+\cdots, \quad j>m,
$$

where $a_{j}$ may be zero. We shall proceed by induction and verify (6) for $m+1$. Evidently $R_{m}=P_{m}^{-1} P P_{m}$ is also an involution, so we have

$$
x=R_{m}^{2}(x)=x+\left((-1)^{j}-1\right) a_{j} x^{j}+\cdots .
$$

Thus $a_{j} \neq 0$ implies that $j$ is even. If we put $Q_{j}(x)=x+b_{j} x^{j}$, then

$$
\begin{aligned}
Q_{j}^{-1} R_{m} Q_{j}(x) & =Q_{j}^{-1}\left(-x+\left(a_{j}-b_{j}\right) x^{j}+\cdots\right) \\
& =-x+\left(a_{j}-b_{j}-(-1)^{j} b_{j}\right) x^{j}+\cdots
\end{aligned}
$$

If $a_{j}=0$, then $R_{m}=R_{\infty}$ and our induction step is complete. If $a_{j} \neq 0$, then $j$ is even, and we can make the coefficient of $x^{j}$ zero by putting $b_{j}=a_{j} / 2$. Hence, our induction step is again complete with $P_{m+1}=P_{m} Q_{j}$. Now $P_{m}$ agrees, with $P_{m+1}$ in all terms of degree $\leqq m$, so our induction process shows us that $P$ is conjugate to $R_{\infty}$ in $\Pi_{\infty}$ as well as in $\Pi_{n}$.

COROLLARY. The normal subgroup of $\Pi_{n}$ generated by any involution different from $P_{0}$ is $I$.

Some simple involutions of $\Pi$ easily come to hand. For each integer $m \geqq 1$ we put

$$
\begin{aligned}
& P_{m}(x)=\frac{x}{\left(1+a_{m} x^{m}\right)^{1 / m}}=x-\left(a_{m} / m\right) x^{m+1}+\cdots, \\
& Q_{m}(x)=\frac{x}{\left(1+b_{m} x^{m}\right)^{1 / m}}=x-\left(b_{m} / m\right) x^{m+1}+\cdots
\end{aligned}
$$


We can easily verify that

$$
\begin{gathered}
P_{m} Q_{m}(x)=\frac{x}{\left(1+\left(a_{m}+b_{m}\right) x^{m}\right)^{1 / m}}, \\
P_{m}^{-1}(x)=\frac{x}{\left(1-a_{m} x^{m}\right)^{1 / m}} .
\end{gathered}
$$

If we put $R_{m}=P_{m}^{-1} R_{\infty} P_{m}$, then

$$
R_{m}(x)=\frac{-x}{\left(1+\left(1-(-1)^{m}\right) a_{m} x^{m}\right)^{1 / m}} .
$$

Evidently $R_{m}$ is an involution. When $m$ is even, we get $R_{m}=R_{\infty}$, and when $m$ is odd, we get

$$
R_{m}(x)=\frac{-x}{\left(1+2 a_{m} x^{m}\right)^{1 / m}}=-x+\left(2 a_{m} / m\right) x^{m+1}+\cdots .
$$

The terms of degree $\leqq n$ in $R_{m}$ give us an involution in $\Pi_{n}$.

6. Special normal subgroups. With each member $h$ of $E(X)$ we associate the polynomials $\pi_{-}(h)=P_{-}$and $\pi_{+}(h)=P_{+}$. Evidently $\pi_{-}$and $\pi_{+}$are homomorphisms from $E^{+}(X)$ into $\Pi^{+}$, and they map $E_{n}^{+}(X)$ onto $\Pi_{n}^{+}$. To see what $\pi_{-}$and $\pi_{+}$do to $E^{-}(X)$, we distinguish two cases:

$$
\begin{array}{lll}
\pi_{-}(g h)=\pi_{-}(g) \pi_{-}(h), & \pi_{+}(g h)=\pi_{+}(g) \pi_{+}(h), & g \in E(X), h \in E^{+}(X), \\
\pi_{-}(g h)=\pi_{+}(g) \pi_{-}(h), & \pi_{+}(g h)=\pi_{-}(g) \pi_{+}(h), & g \in E(X), h \in E^{-}(X) .
\end{array}
$$

One consequence of this is that if $h$ is in $E^{-}(X)$, then

$$
\pi_{-}\left(h^{-1}\right)=\left(\pi_{+}(h)\right)^{-1}, \quad \pi_{+}\left(h^{-1}\right)=\left(\pi_{-}(h)\right)^{-1} .
$$

Another consequence is that if $g$ is in $E^{+}(X)$, and $h$ is in $E^{-}(X)$, then

$$
\begin{aligned}
\pi_{-}\left(h^{-1} g h\right) & =\pi_{+}\left(h^{-1} g\right) \pi_{-}(h)=\pi_{+}\left(h^{-1}\right) \pi_{+}(g) \pi_{-}(h) \\
& =\left(\pi_{-}(h)\right)^{-1} \pi_{+}(g) \pi_{-}(h) .
\end{aligned}
$$

In the same way we get

$$
\pi_{+}\left(h^{-1} g h\right)=\left(\pi_{+}(h)\right)^{-1} \pi_{-}(g) \pi_{+}(h) .
$$

Thus we see that if $g$ is in $E^{+}(X)$, then $\pi_{-}\left(h^{-1} g h\right)$ is conjugate to either $\pi_{-}(g)$ or $\pi_{+}(g)$, depending on whether $h$ is in $E^{+}(X)$ or $E^{-}(X)$. A like result holds for $\pi_{+}\left(h^{-1} g h\right)$. We note that $\pi_{-}$and $\pi_{+}$both map $E_{n}^{-}(X)$ onto $\Pi_{n}^{-}$.

For any subset $\Gamma$ of $\Pi$ we put 


$$
\begin{aligned}
& \Gamma_{-}(X)=\left\{h \in E(X): \pi_{-}(h) \in \Gamma\right\}, \\
& \Gamma_{+}(X)=\left\{h \in E(X): \pi_{+}(h) \in \Gamma\right\},
\end{aligned}
$$

and $\Gamma(X)=\Gamma_{-}(X) \cap \Gamma_{+}(X)$. If $\Gamma$ is a normal subgroup of $\Pi$ that lies in $\Pi^{+}$, then (7) and (8) tell us that $\Gamma(X)$ is a normal subgroup of $\Pi(X)=E(X)$ that lies in $E^{+}(X)$, and that $\Gamma_{-}(X), \Gamma_{+}(X)$, and $\Gamma(X)$ are normal subgroups of $\Pi^{+}(X)=E^{+}(X)$. Of course all these normal subgroups include $\Delta$ or $\Delta(X)$, but we note that $\Delta(X) \supset \Delta^{*}(X)$.

The mapping $\phi$ given by $\phi(G)=\left(\pi_{-}(G), \pi_{+}(G)\right)$ is a one-to-one correspondence between some of the normal subgroups $G$ of $E_{n}^{+}(X)$ that include $A_{n}(X)$ and all of the ordered pairs of normal subgroups from $\Pi_{n}^{+}$. For if we start with an ordered pair $(B, \Gamma)$ of normal subgroups from $\Pi_{n}^{+}$, then we can assign to it the normal subgroup

$$
\bar{\phi}(B, \Gamma)=B_{-}(X) \cap \Gamma_{+}(X)=G
$$

of $E_{n}^{+}(X)$, where $G$ clearly includes $\Delta_{n}(X)$. Since we can always find a member $h$ of $E_{n}^{+}(X)$ with given values of $\pi_{-}(h)$ and $\pi_{+}(h)$ in $\Pi_{n}^{+}$, we have

$$
\phi(\bar{\phi}(B, \Gamma))=\left(\pi_{-}(G), \pi_{+}(G)\right)=(B, \Gamma) .
$$

Thus $\bar{\phi}$ is one-to-one, $\phi$ is onto, and $\phi$ restricted to the range of $\bar{\phi}$ is one-to-one. Similarly, the mapping $\psi$ given by $\psi(G)=\pi_{+}(G)$ is a one-to-one correspondence between some of the normal subgroups $G$ of $E_{n}(X)$ that include $A_{n}(X)$ but lie in $E_{n}^{+}(X)$ and all of the normal subgroups of $\Pi_{n}$ that lie in $\Pi_{n}^{+}$. For if $\Gamma$ is a normal subgroup of $\Pi_{n}$ that lies in $\Pi_{n}^{+}$, then we put $\bar{\psi}(\Gamma)=\Gamma(X)$. Moreover, if $G$ is a normal subgroup of $E_{n}(X)$ that lies in $E_{n}^{+}(X)$, then $\pi_{-}(G)=\pi_{+}(G)$. For both $\pi_{-}(G)$ and $\pi_{+}(G)$ are normal subgroups of $\Pi_{n}^{+}$, and Corollary 2 of Theorem 3 says that they are both normal in $\Pi_{n}$. But (7) and (8) tell us that if $R$ lies in $\Pi_{n}^{-}$, then

$$
R^{-1} \pi_{-}(G) R=\pi_{+}(G) .
$$

Hence, $\pi_{-}(G)=\pi_{+}(G)$.

To see that there are other normal subgroups $G$ of $E_{n}(X)$ that include $A_{n}(X)$ besides the ones mentioned above, we choose a member $g$ of $E_{n}^{+}(X)$ where $\pi_{-}(g)=\pi_{+}(g)=P$, and $P$ lies in $\Pi_{n}^{+}-\Delta_{1}^{* n}$. If we let $\Gamma$ be the group generated by $P$ and $\Delta_{1}^{* n}$, then $\Gamma$ is evidently a normal subgroup of $\Pi_{n}$ that lies in $\Pi_{n}^{+}$. The normal subgroup $G$ of $E_{n}(X)$ generated by $g$ and $\Delta_{1}^{* n}(X)$ evidently lies in $E_{n}^{+}(X)$ and satisfies $\pi_{-}(G)=\pi_{+}(G)=\Gamma$. But for every member $h$ of $G$ we have the same leading coefficient of $\pi_{-}(h)$ and $\pi_{+}(h)$. Hence, $G$ is much smaller than $\Gamma(X)$. 
Lemma 9. Every two involutions in $E(X)$ different from $e$ are conjugate in $S_{-} E(X)$ and also in $S_{+} E(X)$.

Proof. If $h_{i}(i=1,2)$ is such an involution with fixed point $p_{i}$ and satisfying (1) at $p=p_{i}$ with $P=P_{i}$, then $h_{i}^{2}=e, P_{i}^{2}=P_{0}$, and $P_{1}, P_{2}$ are involutions in $\Pi$ different from $P_{0}$. From Lemma 8 we know that

$$
P_{2}=Q^{-1} P_{1} Q=P_{2}^{-1} Q^{-1} P_{1} Q P_{2},
$$

where either $Q$ or $Q P_{2}$ lies in $\Pi^{+}$. Thus $P_{1}$ and $P_{2}$ are conjugate in $\Pi^{+}$, and Lemma 6 tells us that $h_{1}$ and $h_{2}$ are conjugate in $S \_E(X)$ as well as in $S_{+} E(X)$.

\section{THEOREM 4. The commutator subgroup of $E(X)$ is $E^{+}(X)$.}

Proof. Let $h$ be any member of $E^{+}(X)$ and $i$ be any involution of $E(X)$ different from $e$. Our result will follow if we can show that some member $h_{0}$ of the commutator subgroup $C$ of $E(X)$ agrees with $h$ in some neighborhood $U_{+1}$ of $p=+1$ and agrees with $e$ in some neighborhood $U_{-1}$ of $p=-1$. For we have $h=i k i$, where $k$ is in $E^{+}(X)$, and some $k_{0}$ in $C$ agrees with $k$ in some $V_{+1}$ and with $e$ in some $V_{-1}$. Now $k_{1}=i k_{0} i$ agrees with $i k i=h$ in $i\left(V_{+1}\right)=W_{-1}$ and with $i e i=e$ in $i\left(V_{-1}\right)=W_{+1}$. Thus $h_{1}=h_{0}^{-1} k_{1}^{-1} h$ lies in $S E(X)$. Since $C$ is normal in $E(X), k_{1}$ must lie in $C$, and Theorem 1 says that $h_{1}$ lies in $C$. Therefore, $h$ lies in $C$ and $C=E^{+}(X)$. Now our task remains to construct $h_{0}$. We let $p_{0}$ be the fixed point of $i$ and put

$$
f(x)=\left\{\begin{array}{l}
h(x) \text { for } x \text { in some } V_{+1}, \\
i h^{-1} i(x) \text { for } x \text { in some } V_{-1},
\end{array}\right.
$$

and define $f$ in the rest of $X$ so as to make it a member of $E^{+}(X)$ and satisfy $f\left(p_{0}\right)=p_{0}$. Evidently $i$ interchanges the intervals $\left[-1, p_{0}\right]$ and $\left[p_{0},+1\right]$ while $f$ maps each onto itself. Thus

$$
\text { iff } f(x)=\left\{\begin{array}{l}
i\left(i h^{-1} i\right) i h(x)=x \text { for } x \text { in some } W_{+1}, \\
i h i\left(i h^{-1} i\right)(x)=x \text { for } x \text { in some } W_{-1},
\end{array}\right.
$$

and if agrees with some involution $j$ of $E(X)$ different from $e$ in $W_{+1} \cup W_{-1}$. Now Lemma 9 says that $j=g i g^{-1}$ for some $g$ in $E(X)$, and we may assume that $g$ is in $E^{-}(X)$, for if not, then we would take $j g$ and write $j=(j g) i(j g)^{-1}$. Finally, we choose some $g_{0}$ in $E^{-}(X)$ which agrees with $g$ on some $Z_{-1}$ and with $i$ on some $Z_{+1}$. If we put

$$
h_{0}=\left(i g_{0} i g_{0}^{-1}\right)^{-1}\left(i g i g^{-1}\right) \text {, }
$$

then $h_{0}$ evidently lies in $C$. For $x$ in some $U_{+1}$ we have 


$$
\begin{gathered}
i g_{0} i g_{0}^{-1}(x)=i^{4}(x)=x, \\
i g i g^{-1}(x)=i j(x)=i i f(x)=f(x)=h(x),
\end{gathered}
$$

and $h_{0}(x)=h(x)$. For $x$ in some $U_{-1}$ we have

$$
i g_{0} i g_{0}^{-1}(x)=i g i g^{-1}(x) \text {, }
$$

and $h_{0}(x)=x$. This completes the proof.

\section{REFERENCES}

1. D. B. A. Epstein, The simplicity of certain groups of homeomorphisms, Compositio Math., 22 (1970), 165-173.

2. N. J. Fine and G. E. Schweigert, On the group of homeomorphisms of an arc, Ann. of Math., 62 (1955), 237-253.

Received August 27, 1971 and in revised form March 8, 1973.

UNIVERSITY OF BRITISH COLUMBIA 



\section{PACIFIC JOURNAL OF MATHEMATICS}

\section{EDITORS}

RICHARD ARENS (Managing Editor)

University of California

Los Angeles, California 90024

\section{R. A. Beaumont}

University of Washington

Seattle, Washington 98105

\section{J. DuGundJI*}

Department of Mathematics

University of Southern California

Los Angeles, California 90007

D. Gilbarg and J. Milgram

Stanford University

Stanford, California 94305

ASSOCIATE EDITORS
E. F. BECKENBACH
B. H. NeumanN
F. WOLF
K. YoSHIDA

\section{SUPPORTING INSTITUTIONS}

UNIVERSITY OF BRITISH COLUMBIA

CALIFORNIA INSTITUTE OF TECHNOLOGY

UNIVERSITY OF CALIFORNIA

MONTANA STATE UNIVERSITY

UNIVERSITY OF NEVADA

NEW MEXICO STATE UNIVERSITY

OREGON STATE UNIVERSITY

UNIVERSITY OF OREGON

OSAKA UNIVERSITY

\author{
UNIVERSITY OF SOUTHERN CALIFORNIA \\ STANFORD UNIVERSITY \\ UNIVERSITY OF TOKYO \\ UNIVERSITY OF UTAH \\ WASHINGTON STATE UNIVERSITY \\ UNIVERSITY OF WASHINGTON \\ AMERICAN MATHEMATICAL SOCIETY \\ NAVAL WEAPONS CENTER
}

The Supporting Institutions listed above contribute to the cost of publication of this Journal, but they are not owners or publishers and have no responsibility for its content or policies.

Mathematical papers intended for publication in the Pacific Journal of Mathematics should be in typed form or offset-reproduced, (not dittoed), double spaced with large margins. Underline Greek letters in red, German in green, and script in blue. The first paragraph or two must be capable of being used separately as a synopsis of the entire paper. Items of the bibliography should not be cited there unless absolutely necessary, in which case they must be identified by author and Journal, rather than by item number. Manuscripts, in duplicate if possible, may be sent to any one of the four editors. Please classify according to the scheme of Math. Rev. Index to Vol. 39. All other communications to the editors should be addressed to the managing editor, or Elaine Barth, University of California, Los Angeles, California, 90024.

50 reprints are provided free for each article; additional copies may be obtained at cost in multiples of 50 .

The Pacific Journal of Mathematics is issued monthly as of January 1966. Regular subscription rate: $\$ 48.00$ a year (6 Vols., 12 issues). Special rate: $\$ 24.00$ a year to individual members of supporting institutions.

Subscriptions, orders for back numbers, and changes of address should be sent to Pacific Journal of Mathematics, 103 Highland Boulevard, Berkeley, California, 94708.

\section{PUBLISHED BY PACIFIC JOURNAL OF MATHEMATICS, A NON-PROFIT CORPORATION}

Printed at Kokusai Bunken Insatsusha (International Academic Printing Co., Ltd.), 270, 3-chome Totsuka-cho, Shinjuku-ku, Tokyo 160, Japan.

* C. R. DePrima California Institute of Technology, Pasadena, CA 91109, will replace J. Dugundji until August 1974.

Copyright (C) 1973 by

Pacific Journal of Mathematics

All Rights Reserved 


\section{Pacific Journal of Mathematics}

\section{Vol. 49, No. $2 \quad$ June, 1973}

Wm. R. Allaway, On finding the distribution function for an orthogonal polynomial

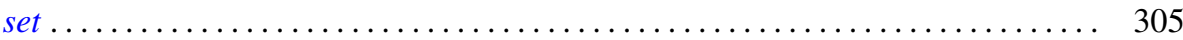

Eric Amar, Sur un théorème de Mooney relatif aux fonctions analytiques bornées... . 311

Robert Morgan Brooks, Analytic structure in the spectrum of a natural system . . . . 315

Bahattin Cengiz, On extremely regular function spaces . . . . . . . . . . . . . . 335

Kwang-nan Chow and Moses Glasner, Atoms on the Royden boundary . . . . . . . . . 339

Paul Frazier Duvall, Jr. and Jim Maxwell, Tame $Z^{2}$-actions on $E^{n} \ldots \ldots \ldots \ldots \ldots . .349$

Allen Roy Freedman, On the additivity theorem for $n$-dimensional asymptotic

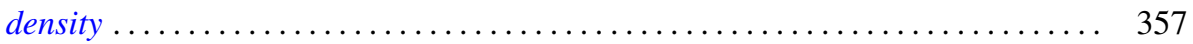

John Griffin and Kelly Denis McKennon, Multipliers and the group $L_{p}$-algebras . . . 365

Charles Lemuel Hagopian, Characterizations of $\lambda$ connected plane continua ....... 371

Jon Craig Helton, Bounds for products of interval functions . . . . . . . . . . . 377

Ikuko Kayashima, On relations between Nörlund and Riesz means . . . . . . . . . . 391

Everett Lee Lady, Slender rings and modules . . . . . . . . . . . . . . . . . . 397

Shozo Matsuura, On the Lu Qi-Keng conjecture and the Bergman representative

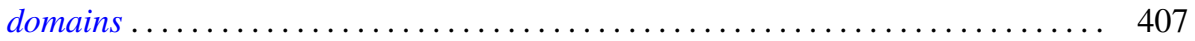

Stephen H. McCleary, The lattice-ordered group of automorphisms of an $\alpha$-set . . . 417

Stephen H. McCleary, o-2-transitive ordered permutation groups .......... 425

Stephen H. McCleary, o-primitive ordered permutation groups. II . . . . . . . . . 431

Richard Rochberg, Almost isometries of Banach spaces and moduli of planar

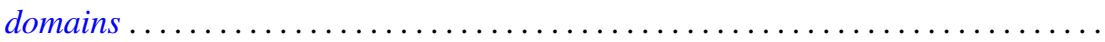

R. F. Rossa, Radical properties involving one-sided ideals . . . . . . . . . . . . . 467

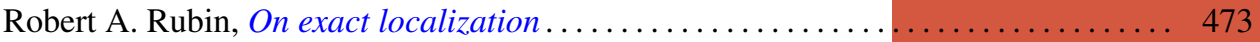

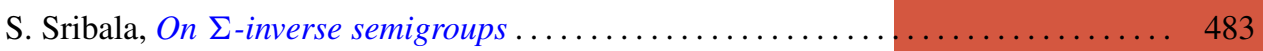

H. M. (Hari Mohan) Srivastava, On the Konhauser sets of biorthogonal polynomials suggested by the Laguerre polynomials ...................... 489

Stuart A. Steinberg, Rings of quotients of rings without nilpotent elements . ...... 493

Daniel Mullane Sunday, The self-equivalences of an $H$-space . . ............ 507

W. J. Thron and Richard Hawks Warren, On the lattice of proximities of $\check{C} e c h$

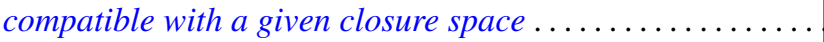

Frank Uhlig, The number of vectors jointly annihilated by two real quadratic forms determines the inertia of matrices in the associated pencil .

Frank Uhlig, On the maximal number of linearly independent real vectors annihilated simultaneously by two real quadratic forms ..............

Frank Uhlig, Definite and semidefinite matrices in a real symmetric matrix pencil . . 561

Arnold Lewis Villone, Self-adjoint extensions of symmetric differential operators . . . 569

Cary Webb, Tensor and direct products . ....................... 579

James Victor Whittaker, On normal subgroups of differentiable

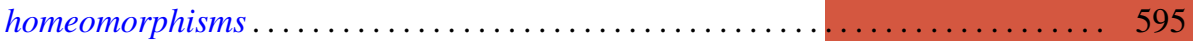

Jerome L. Paul, Addendum to: "Sequences of homeomorphisms which converge to

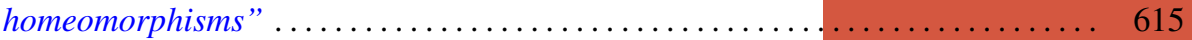

David E. Fields, Correction to: “Dimension theory in power series rings” ........ 616

Peter Michael Curran, Correction to: “Cohomology of finitely presented groups”. . . 617

Billy E. Rhoades, Correction to: “Commutants of some Hausdorff matrices” ...... 617

Charles W. Trigg, Corrections to: "Versum sequences in the binary system” ...... 619 